\title{
Idle and Busy Period Analysis of Two Class Data Traffic through Queueing Technique
}

\author{
Syed Asif Ali Shah \\ Department of Electrical Engineering \\ Mehran University of Engineering and Technology \\ Jamshoro, Pakistan.
}

\author{
Wajiha Shah \\ Department of Electronics Engineering \\ Mehran University of Engineering and Technology, \\ Jamshoro, Pakistan.
}

\begin{abstract}
Analysis of Idle and busy period of any communication system gives the overall information about system behavior when system is empty and data present in the system. In this paper we use a queueing theory approach to model the system with two class data traffic.we develop and analyze the idle and busy period of two class data traffic through queueing system using Markov chain. We also develop the markov chain for calculating the number of customers served during busy period. The length of busy period is also calculated through the construction of Markov chain. The cumulative distribution function of the busy period for each state is also calculated for the various arrival rates.
\end{abstract}

\section{Keywords:}

Idle period, busy period, two class data traffic, queueing system, Markov chain

\section{INTRODUCTION}

The communication systems having various data types are difficult to analyze for their performance. The continuous time systems in which data is continuous mostly analyzed through the differential equations which shows the system behavior. The system behavior through differential equations is not sufficient to understand the overall internal operation of the system. The queueing theory technique can be efficient to analyze these systems to understand their internal behavior. The Markov chain technique provides full information about internal behavior of the system. The Markov chain is constructed with the help of queueing model which shows the all possible system behavior. Idle period and busy period analysis of the system provides information when system is empty and the system having data packets. The idle and busy periods of the system can be analyzed by constructing the Markov chains of these periods through system Markov chain. The length of the busy period in which the total number of data packed served can easily be analyzed by constructing the Markov chain.

The queueing model with two class data [14] have been solved for idle and busy period through Markov chain. The Markov chain for calculating the length of busy period in which data packets served and system is analyzed for their cumulative distribution function

The organization of this paper is as follows: two class data traffic and idle and busy period in Section 2. In Section 3 queueing model of two class data traffic, Markov chain of the system, Markov chain of idle period and Markov chain of busy period are discussed. Finally the results and conclusion are presents in Sections 4 and 5 respectively.

\section{TERMINOLOGIES}

\subsection{Idle Period}

The states of the Markov chain where system is idle or empty is called idle states. Where I represents the set of idle states. Idle period is defined as the time period when there is no customer in the system and server is idle. The idle period starts when last customer of the system leave the system and next customer arrives in the system. The states of the Markov chain where system begins to idle are used to calculate the length of the idle period. The cumulative distribution function of the idle period can be calculated as

$$
\varphi_{\mathrm{i}}(0)=\left\{\begin{array}{c}
1 \text { if } i \text { is in idle state } \\
0 \quad \text { if } i \text { is in busy state }
\end{array}\right\}
$$

The length of the idle period can be calculated as cumulative probability distribution

$$
P(I \leq t)=1-\sum \sigma_{j}^{I} \varphi_{j}(t)
$$

\subsection{Busy Period}

The states of the Markov chain where system is busy is called busy states. Where B represents the set of busy states. Busy period is defined as the time period when there are customer in the system and server is busy. The busy period starts when there is no customer in the system and a customer arrives in the system. The states of the Markov chain where system begins to busy are used to calculate the length of the busy period. The cumulative distribution function of the busy period can be calculated as

$$
\varphi_{\mathrm{i}}(0)=\left\{\begin{array}{cc}
1 & \text { busy state } \\
0 & \text { idle state }
\end{array}\right\}
$$

The length of the busy period can be calculated as cumulative probability distribution

$$
P(I \leq t)=1-\sum \sigma_{j}^{B} \varphi_{j}(t)
$$

\section{SYSTEM MODEL}

\subsection{Queueing Model}

Consider the system which consists of two finite queues in which two class type data are arrives with Poisson distribution $\lambda_{1}$ and $\lambda_{2}$. Both finite queues served by single server with exponential distribution $\mu$. 


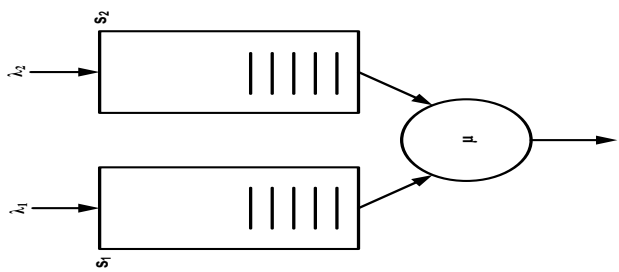

Fig 1: Queueing System

\subsection{Markov Chain}

The system state Markov chain of the system is shown in figure. The states of the Markov chain are represented by two variables $i$ and $j$. The horizontal states of the markov chain represents the overall system behavior of queue1 having one class customer with arrival rate $\lambda_{1}$ and vertical states represents the overall system behavior of queue2 having second class customer with arrival rate $\lambda_{2}$. When there is no customer in the system that system condition is represented by a state $(0,0)$ in the system Markov chain. When there is any arrival occurs in the system either in queue1 or queue2, the system states becomes $(0,1)$ or $(1,0)$. When one of the system queue reached at its maximum capacity the system states becomes $(3,0)$ or $(0,3)$. The system state $(3,3)$ represents the condition when both queues of the system reached at its maximum capacity, the system state

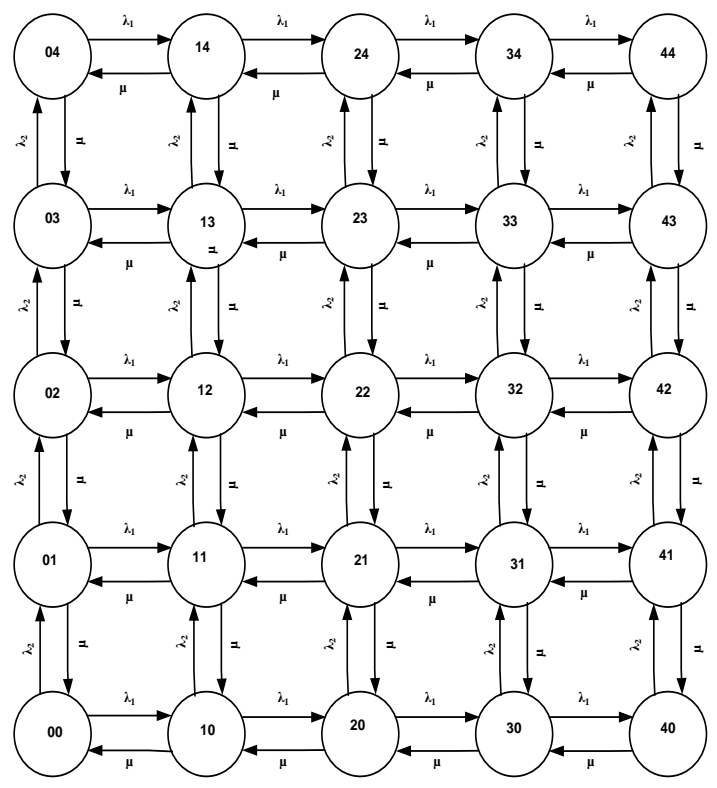

Fig 2: Markov chain

\subsection{Markov Chain of Idle Period}

The length of the idle period of the system is shown in figure. The idle period can easily be represented by constructed the Markov chain of the idle period from the system Markov chain. The Markov chain of the idle period is simply consists on those states of the Markov chain when system is empty. There is only one state in the Markov chain which is $(0,0)$. To construct the Markov chain of idle period by removing the all transitions of the system Markov chain except the transition from $(0,0)$ to $(0,1)$ or $(1,0)$. The idle period of the system is exponentially distributed with $\lambda_{1}$ and $\lambda_{2}$.

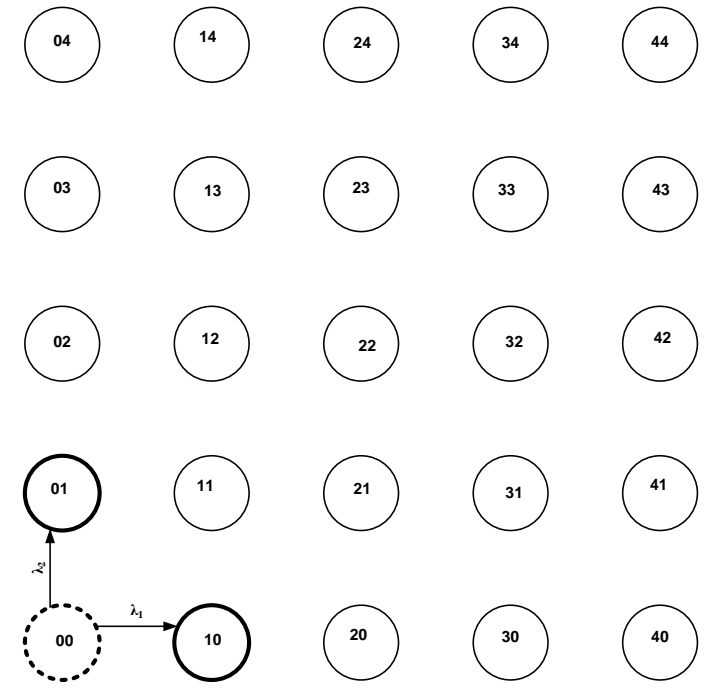

Fig 3: Markov chain of idle period

\subsection{Markov Chain of Busy Period}

The length of the busy period of the system is shown in figure. The busy period can easily represented by constructed the Markov chain of the busy period from the system Markov chain. The Markov chain of the busy period is simply consists on those states of the Markov chain when system is busy. The Markov chain for calculation of length of busy period starts when a system is empty and customer arrives in the system.

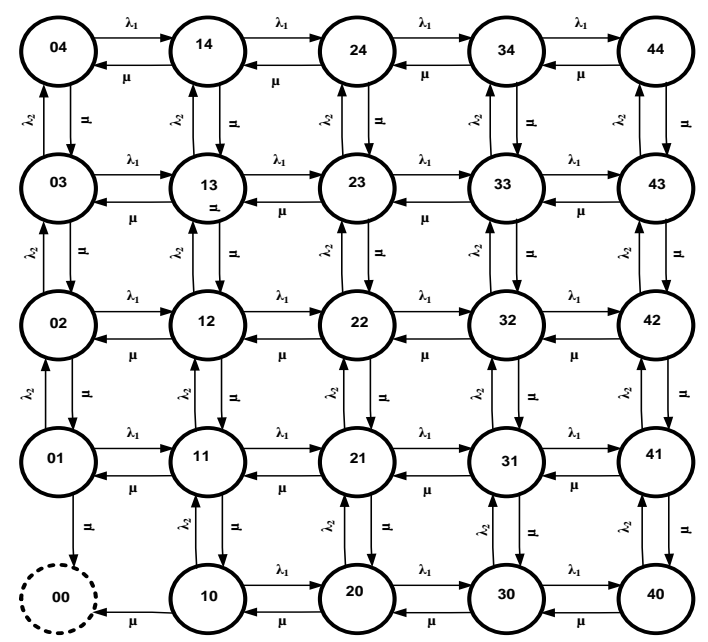

Fig 4: Markov chain of busy period

\subsection{Markov Chain of Length of Busy \\ Period}

The Markov chain for calculation of the probability distribution of the number of customers served in a busy period is shown in figure. T he Markov chain of the length of the busy period is constructed through the system state and counting process Markov chain. The system states and counting process are represented by the horizontal and vertical states of the length of the busy period s Markov chain respectively. The busy period of the system in which customers are served is represented by inclined states of busy period Markov chain. There are three types of states in the busy state Markov chain. These states are starting, indirect and absorbing states. Starting states are those states where busy period begins. Indirect states are those where system is already in busy period and new customer arrives in the system. The absorbing states are those where busy period is completed and system becomes idle. 


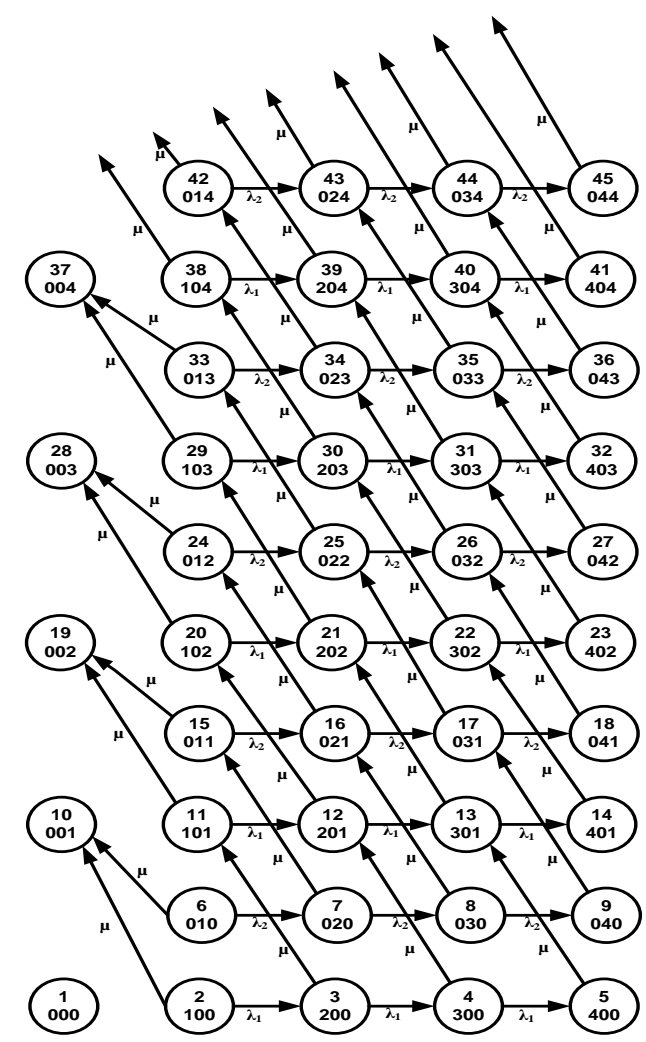

Fig 5: Markov chain of length of busy period to calculate the number of customers served during busy period

\section{RESULTS AND DISCUSSION}

The simulation and analytical program for calculation of the cumulative distribution function of number of customers served in a busy period are developed in MATLAB and $\mathrm{VC}++$.

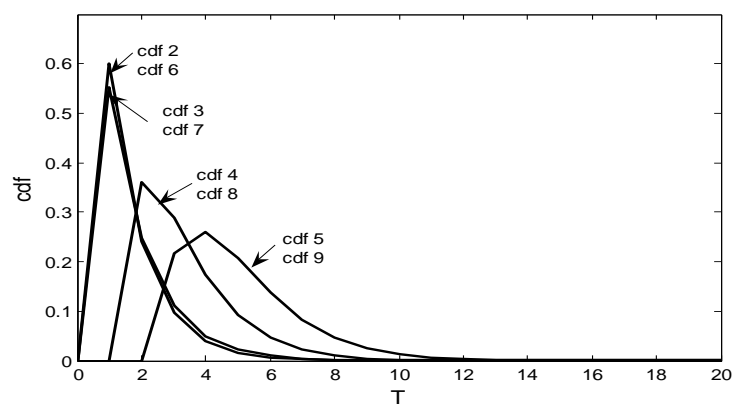

Fig 6: Cumulative distribution function of each state for $\lambda_{1}=0.6, \lambda_{2}=0.2$, and $\mu=0.4$.

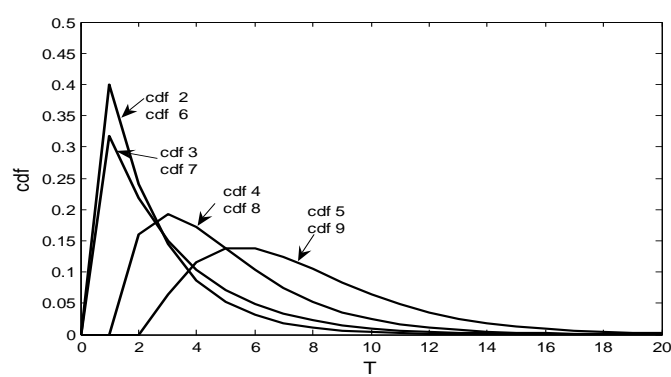

Fig 7: Cumulative distribution function of each state for $\lambda_{1}=0.4, \lambda_{2}=0.6$, and $\mu=0.4$.
Fig. 6 shows the cumulative distribution function of each state where busy period begins for values of arrival and service rates, $\lambda_{1}=0.6, \lambda_{2}=0.2$ and $\mu=0.4$. It shows the length of the busy period that the customer served during busy period. The busy period is shorter when there is an one customer in the system which is described by the cdf of states (2) and (6). As the number of customers increases in the system, the busy period is becomes larger until all customers presents in the system served and then system is become idle. These states are represented by cdf of states (3), (7), (4), (8), (5) and (9). Fig. 7 shows the same behavior for the values of, $\lambda_{1}=0.4, \lambda_{2}=0.6$ and $\mu=0.4$.

\section{CONCLUSION}

In this paper, we used a queueing theory approach to model the system with two class data traffic. The system is solved using idle and busy period Markov chain to analyze the system behavior at empty and busy states. Markov chain for the calculation of the length of busy period period is also developed and analyzed. The cumulative distribution function of the busy period for each state is analyzed and results was discussed.

\section{ACKNOWLEDGMENTS}

The authors are thankful to Mehran University of Engineering \& Technology, Jamshoro, Pakistan for providing necessary research facilities during their research work.

\section{REFRENCES}

[1] Latouche, G., and Ramswami, V.,. 1993. A logarithmic reduction algorithm for quasi-birth-death processes, J. Appl. Prob., vol. (30): 650 -674.

[2] Shah, Wajiha., 2010. Performance Modeling of Queueing Systems using Matrix Geometric Method, $\mathrm{Ph} . \mathrm{D}$. Thesis, Faculty of Electrical Engineering and Information Technology, Vienna University of Technology, Wien, Austria, April..

[3] Asif A.S.S. 2010. Flow Time Analysis of An Early Arrival System Using Discrete time Hypogeometrical Distribution, AMS 2010, Kota Kinabalu, Malaysia.

[4] Bocharov, P. P. 1996. Stationary Distribution of a Finite Queue with Recurrent Flow and Markov Service, Avtom. Telemekh., Vol. (9): 66-78.

[5] B. Van Houdt and C. Blondia,. 2004. The waiting time distribution of a type $k$ customer in a MAP $[K] / P H[K] / C$ $(c=1,2)$ queue using $Q B D$, Stochastic Models, Vol. 20, pp. $55-69$.

[6] Gupta, U.C., Samanta, S.K., and Sharma, R.K... 2000. Computing queueing length and waiting time distributions in finite-buffer discrete-time multi-server queues with late and early arrivals, Computers and Mathematics with Applications, vol. (48): 1557- 1573.

[7] Bocharov, P. P. 1996. Stationary Distribution of a Finite Queue with Recurrent Flow and Markov Service, Avtom. Telemekh., No. 9, 66-78.

[8] Thomas G. Robertazzi. Computer Networks and Systems Queueing Theory and Performance Evaluation. Springer Verlag, New York, 1994. 